\title{
GENERIC ONE-PARAMETER FAMILIES OF VECTOR FIELDS ON TWO-DIMENSIONAL MANIFOLDS
}

\author{
BY J. SOTOMAYOR ${ }^{1}$ \\ Communicated by S. Smale, November 30, 1967
}

Introduction. This paper is an announcement of a study on the theory of the topological variation of the phase space of one-parameter families of vector fields (ordinary differential equations, flows). This theory, sometimes called bifurcation theory, has been developed since $\mathrm{H}$. Poincaré from several points of view; see, for example, $[1],[2],[3],[4],[5]$. Here, we will be mainly interested in a collection of one-parameter families of vector fields which has the following properties: (a) it is large with respect to all the families, and (b) its elements exhibit a topological variation which is amenable to simple description.

Collections with properties (a) and (b) are currently called "generic" and were introduced in the qualitative global analysis of differential equations by M. Peixoto [6], S. Smale [8], and I. Kupka [11]. See S. Smale [9] for a thorough monography on this field.

The geometry of the set $\Sigma$ of structurally stable vector fields and the study of "generic" one-parameter families of vector fields are closely related. A vector field is structurally stable if its phase space does not change topologically under small perturbations; a oneparameter family of vector fields exhibits: the simpler a phase-space topological variation, the larger the intersection it has with $\Sigma$, or equivalently, the smaller the intersection it has with its complement - the set of nonstructurally stable vector fields.

The importance of the set of nonstructurally stable vector fields for the study of the topological variation of the phase space of vector fields was noticed by A. Andronov and E. Leontovich. In [12] they defined the concept of first-order structural stability as a possible guide to pursue such study.

In compact two-dimensional manifolds, the only case considered here, the set of first-order structurally stable vector fields is an imbedded Banach manifold of class $C^{1}$ and codimension one of the Banach manifold of vector fields; see [13]. This fact, although (as follows from [14]) not sufficient to describe completely the "generic"

1 This work was done with the partial support of the National Science Foundation, Grant GP-5603. 
one-parameter families of vector fields, motivates the main result of this paper: There is an immersed Banach submanifold, $\Sigma_{1}$, of class $C^{1}$ and codimension one of the Banach manifold of vector fields with $C^{r}$-topology, $r \geqq 4$, such that the "generic" one-parameter families of vector fields intersect it transversally at points where they are not vector fields of Kupka-Smale type. This result is stated in a precise and complete form in Theorems 1 and 2 ; it answers questions raised by M. Peixoto.

For the proof of these theorems, which will be published elsewhere, the characterization of $\Sigma$ given by M. Peixoto [7] and the approximation techniques of I. Kupka and S. Smale, [11], [10], are essential.

Preliminary definitions. Let $M^{2}$ be a $C^{\infty}$ two-dimensional compact manifold. Call $\mathfrak{X}^{r}$ the space of $C^{r}$-tangent vector fields defined on $M^{2}$ under the $C^{r}$-topology. Denote by $\phi_{X}: R \times M^{2} \rightarrow M^{2}$ the $C^{r}$-flow induced by $X \in \mathfrak{X}^{r}$.

Definition 1 . Let $X$ and $Y \in \mathfrak{X}^{r}, r \geqq 1$; they are said to be topologically equivalent if there is a homeomorphism of $M^{2}$ onto itself sending trajectories of $X$ onto trajectories of $Y$. If $X$ has a neighborhood $N(X)$ in $\mathfrak{X}^{r}$ such that it is topologically equivalent to every $y \in N(X)$, $X$ is said to be structurally stable.

Denote by $\Sigma^{r}$ the set of structurally stable vector fields and denote by $\mathfrak{X}_{1}^{r}$ its complement relative to $\mathfrak{X}^{r}$ endowed with the induced $C^{r}$. topology.

Definition 2. A continuous function $\xi: J=[a, b] \rightarrow \mathfrak{X}^{r}$ is called a one-parameter family of vector fields. A point $\lambda_{0} \in J$ is said to be an ordinary value of $\xi$ if for any $\epsilon>0$, it has a neighborhood $N\left(\lambda_{0}\right)$ such that $\xi(\lambda)$ is topologically equivalent to $\xi\left(\lambda_{0}\right)$ for every $\lambda \in N\left(\lambda_{0}\right)$; $\lambda_{0}$ is called a bifurcation value of $\xi$ if it is not an ordinary value of $\xi$.

Quasi-generic vector fields. Let $p \in M^{2}$ be a singular point of $X \in \mathfrak{X}^{r}$, i.e. $X(p)=0$. For $r \geqq 1$ and any $V \in \mathfrak{X}^{r},[V, X](p)$ depends only on $V(p)$. This remark makes it possible to define an endomorphism $L_{p}$ of the tangent space $T_{p}$ at $p . L_{p}$ is defined as follows: if $v \in T_{p}$, let $V \in \mathfrak{X}^{r}$ be any extension of it and define $L_{p}(v)=[V, X](p)$. The determinant and trace of $L_{p}$ are denoted respectively by $\Delta(X, p)$ and $\sigma(X, p)$. If $L_{p}$ is an isomorphism, the singular point $p$ of $X$ is said to be simple. It is said to be generic if the eigenvalues of $L_{p}$ have nonvanishing real parts; if the eigenvalues of $L_{p}$ are real and have opposite sign, $p$ is called a saddle; if they have equal sign, $p$ is called a node; if the eigenvalues have nonvanishing imaginary parts, $p$ is called a focus. 
Let $\lambda_{1}$ and $\lambda_{2}$ be the eigenvalues of $L_{p}$, assume that they are real and different and denote respectively by $T_{1}$ and $T_{2}$ the eigenspaces associated with them. Call respectively $\pi_{1}$ and $\pi_{2}$ the projections of $T_{p}$ onto $T_{1}$ and $T_{2}$ associated with the splitting $T_{p}=T_{1} \oplus T_{2}$.

DEFINITION 3. A singular point $p$ of $X \in \mathfrak{X}^{r}, r \geqq 2$, is said to be a saddle-node if only one of the eigenvalues of $L_{p}$, say $\lambda_{1}$, vanishes and $\Delta_{1}(X, p, v) \neq 0, v \in T_{1}, v \neq 0, \Delta_{1}(X, p, v) v=\pi_{1}[V,[V, X]](p)$ where $V$ is any $C^{r}$-extension of $v$. It can be proved that the saddle-node is well defined.

Suppose that the eigenvalues of $L_{p}$ have nonvanishing imaginary parts. Let $I$ be a $C^{\infty}$ arc on $M^{2}$ with one extreme at $p$. It is classical that the flow $\phi_{X}$ defines homeomorphism $\rho_{X}: I_{0} \rightarrow I$ on an $I$-neighborhood $I_{0}$ of $p ; \rho_{X}$ assigns to $q \in I_{0}$ the point where the semiorbit $\phi_{X}(t, q), t>0$, meets $I$ for the first time; $\rho_{X}$ is of class $C^{r}$ if $X$ is so.

Definition 4. (a) A singular point $p$ of $X \in \mathfrak{X}^{r}, r \geqq 3$, is said to be a composed focus if $\rho_{X}^{1}(p)=1$ and $\rho_{X}^{3}(p) \neq 0$.

(b) A singular point $p$ of $X \in \mathfrak{X}^{r}, r \geqq 3$, is said to be quasi-generic if it is either a saddle-node (Definition 3 ) or a composed focus.

Suppose that $X$ has a nontrivial periodic orbit through $p$ and let $I$ be a $C^{\infty}$ arc transversal to $X$ which has $p$ as interior point. The flow $\phi_{X}$ defines a homeomorphism $\pi_{X}: I_{0} \rightarrow I$ defined on an $I$-neighborhood $I_{0}$ of $p ; \pi_{X}(q)$ is the point where the semiorbit $\phi_{X}(t, q), t>0$, meets $I$ for the first time. $\pi_{X}$ is of the same class of differentiability as $X$ and is currently called the Poincare transformation associated with $X$, $p$, and $I$.

Definition 5. A periodic orbit of $X \in \mathfrak{X}^{r}, r \geqq 1$, passing through $p$ is called generic if $\left|\pi_{X}^{1}(p)\right| \neq 1$; it is called quasi-generic if either $\pi_{X}^{1}(p)=1, r \geqq 2$, and $\pi_{X}^{(2)}(p) \neq 0$ or $\pi_{X}^{1}(p)=-1, r \geqq 3$, and $\left(\pi_{X} \circ \pi_{X}\right)^{(3)}(p) \neq 0$.

Denote by $\omega_{X}(p)$ (resp. by $\alpha_{X}(p)$ ) the $\omega$-limit (resp. the $\alpha$-limit) set of the orbit of $X$ which passes through $p$.

DEFINITION 6. (a) If in every neighborhood of $p$ there are points $q$ such that $\omega_{X}(q) \neq \omega_{X}(p)$ (resp. $\alpha_{X}(q) \neq \alpha_{X}(p)$ ), then the orbit of $X$ passing through $p$ is called $\omega$ separatrix (resp. $\alpha$ separatrix).

(b) An $\alpha$ and (or) $\omega$ separatrix is said to be a saddle connection (saddle separatrix) if its $\alpha$ and (or) $\omega$-limit sets are saddle or saddlenode singular points.

(c) If the $\alpha$ and $\omega$-limit sets of a saddle connection of $X$ are equal to a saddle point $q$, such that $\sigma(X, q) \neq 0$, then the set formed by the saddle connection and $q$ is called a simple graph.

Definition 7. Assume $r \geqq 3$. (a) $Q_{1}^{r}$ is defined to be the set of $C^{r}$ vector fields which have one quasi-generic singular point, have only generic periodic orbits, and do not have saddle connections. 
(b) $Q_{2}^{r}$ is defined to be the set of $C^{r}$-vector fields which have one quasi-generic periodic orbit as unique nongeneric periodic orbit, have only generic singular points, and do not have saddle connections.

(c) $Q_{3}^{r}$ is defined to be the set of $C^{r}$-vector fields which have one saddle connection which, in case of being a self-connection is part of a simple graph, have only generic singular points and generic periodic orbits.

(d) [K-S ] (Kupka-Smale vector fields) is defined to be the set of $C^{r}$-vector fields which have only generic singular points, only generic periodic orbits, and do not have saddle connections.

(e) $P^{r}$ is defined to be the set of $C^{r}$-vector fields $X$ for which $\omega_{X}(p)$ and $\alpha_{X}(p)$, for every $p \in M^{2}$, can only be singular points, periodic orbits, or graphs, i.e. vector fields for which Poincaré-Bendixon theorem is valid.

Banach submanifolds. Let $B$ be a Banach manifold of class $C^{\infty}$ in the sense of S. Lang [15, p. 16]; obviously, $\mathfrak{X}^{r}$ belongs to this category.

Definition 8. (a) A subset $S \subset B$ is said to be an imbedded Banach manifold of class $C^{3}$ and codimension $k$ of $B$ if every $p \in S$ has a neighborhood $N(p)$ such that there exists a $C^{s}$-function $f: N(p) \rightarrow R^{k}$ such that $d f_{p}$ has maximum rank and $f^{-1}(0)=S \cap N(p)$.

(b) $S \subset B$ is said to be an immersed Banach manifold of class $C^{\circ}$ and codimension $k$ of $B$ if there is a countable family $\left\{S_{i}\right\}_{i \in N}$ of imbedded Banach manifolds of class $C^{s}$ and codimension $k$ of $B$ such that $S_{i} \subset S_{i+1}, i \in N$, and $S=\bigcup_{i=1}^{\infty} S_{i}$.

It is easily verified that Definition 7 implies the definitions of the same concepts, as given in [13, p. 19 and 20].

Definitron 9. $\Phi^{r}$ is defined to be the set of one parameter families of vector fields $\xi: J \rightarrow \mathfrak{X}^{r}$ of class $C^{1}$, under the $C^{1}$-topology. Obviously, $\Phi^{r}$ is a Banachable space.

The results. We assume $r \geqq 4$.

THEOREM 1. (a) $\Sigma_{1}^{r}=\operatorname{Pr}^{r} \cap\left(\bigcup_{i=1}^{3} Q_{i}^{r}\right)$ is an immersed Banach manifold of class $C^{1}$ and codimension one of $\mathfrak{X}^{r}$.

(b) $\Sigma_{1}^{r}$ is dense in $\mathfrak{X}_{1}^{r}$.

(c) If $X \in \Sigma_{1}^{r}$, there is a $\Sigma_{1}^{r}$-neighborhood $N(X)$ of $X$ such that $X$ is topologically equivalent to every $Y \in N(X)$.

THEOREM 2. Let $\Gamma^{r}$ be the set of one-parameter families of vector fields $\xi \in \Phi^{r}$ such that

(a) $\xi(J) \subset \Sigma_{1}^{r} \cup[K-S]^{r}$.

(b) $\xi$ is transversal to $\Sigma_{1}^{r}$.

(c) The set of bifurcation values of $\xi$ is a closed nowhere dense set of $J$; it coincides with $J-\xi^{-1}\left(\Sigma^{r}\right)$. 
Then, $\Gamma^{r}$ contains a residual subset of $\Phi^{r}$; in particular, it is a dense subset of $\Phi^{r}$.

\section{REFERENCES} 15-31.

1. G. F. Duff, Limut cycles and rotated vector fields, Ann. of Math. (2) 57 (1953),

2. M. Urabe, Infinitesimal deformation of cycles, J. Sci. Hiroshima University, A 18(1954), 37-53.

3. A. Andronov and C. Chaikin, Theory of oscillations, Chapter VI, Princeton Univ. Press, Princeton, N. J., 1945.

4. L. Marcus and A. Aeppli, Integral equivalence of vector fields on manifolds and bifurcation of differential systems, Amer. J. Math. 85 (1963), 633-654.

5. R. Sacker, Invariant surfaces and bifurcation of periodic solutions of ordinary differential equations, New York University, Courant Inst. Math. Sci. Tech. Rep. IMM-NYU 333, 1964.

6. M. Peixoto, On structural stability, Ann. of Math. (2) 69(1959), 199-222.

7. - Structural stability on two dimensional manifolds, Topology 1 (1962), 101-120.

8. S. Smale, On dynamical systems, Bol. Soc. Mat. Mexicana (2) 5 (1960), 195-198.

9. —_, Differentiable dynamical systems, Bull. Amer. Math. Soc. 73 (1967), 747-817.

10. Stable manifolds for differential equations and diffeomorphisms, Ann. Scuola Norm. Sup. Pisa, Serie III 17 (1963), 97-116.

11. I. Kupka, Contribution a la théorie des champs generiques, Cont. to Diff. Eq. 2 (1963), 451-484.

12. A. Andronov and R. Leontovich, Sur la théorie de la variation de la structure qualitative de la division du plan en trajectoires, Dokl. Akad. Nauk. 21 (1938), 427-430.

13. J. Sotomayor, Estabilidade estrutural de primeira ordem e variedades de Banach, Thesis I.M.P.A., Rio de Janeiro, 1964.

14. - On certain one-parameter family of vector fields, Notas de Matemáticas, Ins. Matemáticas, Univ. Ingenieria, Lima, 1965.

15. S. Lang, Introduction to differentiable manifolds, Interscience, New York, 1962.

UNIVERSity of CALIFoRNiA, BERKELEY, AND

UNIVERSIDAD DE INGENIERIA, LIMA 